\title{
Nucleonic Direct Urca Processes and Cooling of the Massive Neutron Star by Antikaon Condensations
}

\author{
Yan Xu $\mathbb{D}^{1,2}$ Wen Bo Ding, ${ }^{3}$ Cheng Zhi Liu, ${ }^{2}$ and J. L. Han ${ }^{1}$ \\ ${ }^{1}$ National Astronomical Observatories, Chinese Academy of Sciences, DaTun Road A1, Chaoyang District, Beijing 100101, China \\ ${ }^{2}$ Changchun Observatory, National Astronomical Observatories, Chinese Academy of Sciences, Changchun 130117, China \\ ${ }^{3}$ College of Mathematics and Physics, Bohai University, Jinzhou 121000, China
}

Correspondence should be addressed to Yan Xu; xuy@cho.ac.cn

Received 21 August 2020; Revised 21 September 2020; Accepted 30 September 2020; Published 17 October 2020

Academic Editor: Muhammad Farasat Shamir

Copyright (c) 2020 Yan Xu et al. This is an open access article distributed under the Creative Commons Attribution License, which permits unrestricted use, distribution, and reproduction in any medium, provided the original work is properly cited.

\begin{abstract}
Nucleonic direct Urca processes and cooling of the massive neutron stars are studied by considering antikaon condensations. Calculations are performed in the relativistic mean field and isothermal interior approximations. Neutrino energy losses of the nucleonic direct Urca processes are reduced when the optical potential of antikaons changes from -80 to $-130 \mathrm{MeV}$. If the center density of the massive neutron stars is a constant, the masses taper off with the optical potential of antikaons, and neutrino luminosities of the nucleonic direct Urca processes decrease for $\rho_{\mathrm{CN}}=0.5 \mathrm{fm}^{-3}$ but first increase and then decrease for larger $\rho_{\mathrm{CN}}$. Large optical potential of antikaons results in warming of the nonsuperfluid massive neutron stars. Massive neutron stars turn warmer with the protonic ${ }^{1} S_{0}$ superfluids. However, the decline of the critical temperatures of the protonic ${ }^{1} S_{0}$ superfluids for the large optical potential of antikaons can speed up the cooling of the massive neutron stars.
\end{abstract}

\section{Introduction}

Just two years after Chadwick discovered the neutron [1], Baade and Zwicky suggested the possibility of the existence of neutron stars (NSs) in 1934 [2]. NSs were confirmed by the discovery of the first radio pulsar named PSR B1919+ 211 in 1967 [3]. NSs shown as pulsars are one of the most intriguing observational objects owing to special characteristics of themselves. The unknown core compositions of NSs is one of the hot topics that need to be solved with knowledge from several fields: high-energy astrophysics, nuclear physics, superfluid hydrodynamics, neutrino physics, hadronic physics, general relativity, and so on. After decades of observational, experimental, and theoretical studies, the equation of states (EOSs) and global properties of extremely dense matter in cores of NSs are mysterious. The EOSs and NS observables, such as the gravitational mass $M$ and radius $R$, can be connected through the Tolman-Oppenheimer-Volkoff (TOV) equations $[4,5]$. The precise mass measurements of the massive pulsars in binary star systems are the best and most direct constraint on the
EOSs. At present, the three most massive pulsars, PSRs J1614-2230 with a mass of $M=1.97 \pm 0.04 M_{\odot}, \mathrm{J} 0348+0432$ with a mass of $M=2.01 \pm 0.04 M_{\odot}$, and $\mathrm{J} 0740+6620$ with a mass of $M=2.14_{-0.09}^{+0.10} M_{\odot}$, have put a reasonable limitation on EOSs of NSs [6-8]. The maximum masses of NSs should be $>2.0 M_{\odot}$ [9].

Observational data of the surface thermal radiation of pulsars as an indication of NS cooling can also constrain NS structure. Stabler firstly assessed NS thermal emission in 1960 [10]. Chiu analyzed the probability of discovering NSs through their thermal emission in 1964 [11]. Computations of NS cooling were carried out in 1964 and 1965 [12-14]. Tsuruta and Cameron presented the basis of the rigorous NS cooling theory in 1966, one year prior to the discovered pulsars. The cooling processes can have three stages: the internal relaxation stage ( $t \leqslant 50$ years), neutrino cooling stage with isothermal interior $\left(t \leqslant 10^{5}-10^{6}\right.$ years), and photon cooling stage $\left(t \geq 10^{5}-10^{6}\right.$ years) governed by photon surface emission [15-17].

However, detected thermal surface radiation from cooling isolated NSs is at the neutrino cooling stage. 
Therefore, we focus on neutrino cooling in the work. The main cooling regulators at this stage are as follows: (1) a strong neutrino emission from a NS core which controls NS cooling rate and (2) the suppression of the baryonic superfluids on neutrino emission. Nucleonic direct Urca processes are expected to largely affect the cooling of NSs because they are the most powerful neutrino emission in NS interior and closely related to the protonic superfluids. The generation of proton-proton (pp) Cooper pair under the attractive interaction helps protons to be paired through the Bardeen-Cooper-Schrieffer (BCS) theory [18, 19]. Unlike the neutronic ${ }^{1} S_{0}$ superfluids, the protonic ${ }^{1} S_{0}$ superfluids can occur in the core of a NS, where it is highly relevant to the nucleonic direct Urca processes.

In 1986, Kaplan and Nelson pointed out that $K^{-}$mesons can form the Bose-Einstein condensed phases in dense nuclear matter [20]. Antikaon condensed phases can soften the EOSs, change the whole properties of NSs [21-30], and significantly improve the protonic ${ }^{1} S_{0}$ superfluids in a NS core [31]. We now investigate how a NS cooling rate changes if the protonic ${ }^{1} S_{0}$ superfluids appear in the massive NS with antikaon condensed phases.

In Section 2, we discuss the mass ranges of the appearance of the Bose-Einstein condensates in NS matter, neutrino emissivities, and the corresponding neutrino luminosities of the nucleonic direct Urca processes, the relationship between cooling curves and the protonic ${ }^{1} S_{0}$ superfluids based on the approximations of the relativistic mean field (RMF), and isothermal NS interior. In Section 3, we present the numerical results of how $K^{-}$and $\bar{K}^{0}$ condensations affect neutrino emissivities, the corresponding neutrino luminosities of the nucleonic direct Urca processes, and speed of NS cooling. The summary is given in Section 4.

\section{Theoretical Framework}

2.1. The RMF Theory. The RMF model and the corresponding effective Lagrangian for the nucleonic and leptonic parts have been clearly described with detailed references [31-36]. Here, we expand the Lagrangian including the antikaons. The Lagrangian for the antikaonic part in the minimal coupling scheme could be written as follows [28]:

$$
L_{K}=D_{\mu}^{*} \overline{\mathrm{K}} \mathrm{D}^{\mu} K-m_{K}^{*} \overline{\mathrm{K}} \mathrm{K}
$$

where antikaons are $\bar{K} \equiv K^{-}$or $\bar{K}^{0}$ and $m_{K}^{*}$ is the effective mass of (anti)kaons as follows:

$$
m_{K}^{*}=m_{K}-g_{\sigma K} \sigma,
$$

where $m_{K}(=495 \mathrm{MeV})$ expresses the bare mass of (anti) kaons and $g_{\sigma K}$ denotes the isoscalar scalar $\sigma$ meson-kaon coupling. The vector fields are coupled to antikaons similar to the minimal coupling scheme through the following relationship:

$$
D_{\mu}=\partial_{\mu}+i g_{\omega K} \omega_{\mu}+i g_{\rho K} \tau_{K} \cdot \boldsymbol{\rho}_{\mu}
$$

where $\sigma, \omega_{\mu}$, and $\rho_{\mu}$ represent isoscalar scalar, isoscalar vector, and isovector vector fields, respectively, and $g_{\omega K}$ and $g_{\rho K}$ represent $\omega$ meson-kaon and $\rho$ meson-kaon couplings, respectively.

The equation of motion for the antikaonic part from the Lagrangian equation (1) can be expressed through solving the Euler-Lagrange equation:

$$
\left[D_{\mu} D^{\mu}+m_{K}^{* 2}\right] K=0 .
$$

The dispersion relation of s-wave $(\mathbf{k}=0)$ condensation for antikaons $\bar{K}$ representing the in-medium energies can be given by means of the mathematic deduction:

$$
\omega_{K^{-}, \bar{K}^{0}}=m_{K}-g_{\sigma K} \sigma-g_{\omega K} \omega_{0} \mp \frac{1}{2} g_{\rho K} \rho_{03} .
$$

It changes linearly in the meson fields. In the RMF approximation, $\sigma=\langle\sigma\rangle, \omega=\left\langle\omega_{0}\right\rangle$, and $\rho=\left\langle\rho_{03}\right\rangle$ are the expectation values of $\sigma$, $\omega$, and $\rho$ meson fields in NS matter. Moreover, $\rho_{03}=\rho_{p}-\rho_{n}$, where $\rho_{n}$ and $\rho_{p}$ represent the neutronic and protonic densities, respectively. The isospin projections $I_{3 \bar{K}}=-1 / 2$ for meson $K^{-}$and $I_{3 \bar{K}}=1 / 2$ for meson $\bar{K}^{0}$ are clearly written in (5), respectively.

The source terms should be added for the meson field equations if $K^{-}$and $\bar{K}^{0}$ condensed phases are considered in NS matter. The scalar and vector densities of antikaons are same for s-wave antikaon condensed phases, using the dispersion relation equation (5) and RMF approximation, given by

$$
\begin{aligned}
\rho_{K^{-}, \bar{K}^{0}} & =2\left(\omega_{K^{-}, \bar{K}^{0}}+g_{\omega K} \omega_{0} \pm \frac{1}{2} g_{\rho K} \rho_{03}\right) \overline{\mathrm{K} K} \\
& =2 m_{K}^{*} \overline{\mathrm{K}} \mathrm{K} .
\end{aligned}
$$

The meson field equations are then simplified to

$$
\begin{gathered}
m_{\sigma}^{2} \sigma=g_{\sigma N}\left[\rho_{\mathrm{SN}}-\frac{g_{2}}{g_{\sigma N}} \sigma^{2}-\frac{g_{3}}{g_{\sigma N}} \sigma^{3}\right]+g_{\sigma K}\left(\rho_{K^{-}}+\rho_{\bar{K}^{0}}\right), \\
m_{\omega}^{2} \omega_{0}=g_{\omega N}\left(\rho_{n}+\rho_{p}\right)-g_{\omega K}\left(\rho_{K^{-}}+\rho_{\bar{K}^{0}}\right), \\
2 m_{\rho}^{2} \rho_{03}=g_{\rho N} \rho_{03}-g_{\rho K}\left(\rho_{K^{-}}-\rho_{\bar{K}^{0}}\right),
\end{gathered}
$$

where $\rho_{\mathrm{SN}}$ is the nucleonic scalar density [22], in which $N \equiv(n, p)$.

The total energy density $\varepsilon$ comes from the contributions of nucleons, leptons, and antikaons. The energy density of nucleons plus leptons has been described in detail in references $[32,33]$. The energy density of antikaonic part is given by

$$
\begin{aligned}
\mathcal{E}_{\bar{K}} & =2 m_{K}^{*} \bar{K} K \\
& =m_{K}^{*}\left(\rho_{K^{-}}+\rho_{\bar{K}^{0}}\right) .
\end{aligned}
$$

Because antikaon condensation does not directly contribute to the pressure, the expression of pressure remains the same $[22,37]$. However, the pressure can be indirectly changed through the changes of the meson field equations 
(7)-(9) due to the additional source terms for antikaon condensation.

In the interior of NSs, nucleons and leptons undergo usual $\beta$-decay processes, namely, the nucleonic direct Urca processes $n \longrightarrow p+l+\bar{\nu}_{l}$ and $p+l \longrightarrow n+v_{l}$. As the Fermi momenta of nucleons increase, the strangeness changing processes may happen which are $N \rightleftharpoons N+\bar{K}$ and $e^{-} \rightleftharpoons K^{-}+v_{e}$. Hence, the chemical potentials of nucleons, leptons, and antikaons fulfill the following chemical equilibrium conditions:

$$
\begin{aligned}
\mu_{n}-\mu_{p} & =\mu_{e}=\mu_{K^{-}}, \\
\mu_{\bar{K}^{0}} & =0 .
\end{aligned}
$$

The antikaon condensation occurs in NS matter if the inmedium energies $\omega_{\bar{K}}$ are equivalent of the chemical potentials $\mu_{\bar{K}}$ :

$$
\begin{aligned}
& \omega_{K^{-}}=\mu_{K^{-}}=\mu_{e}, \\
& \omega_{\bar{K}^{0}}=\mu_{\bar{K}^{0}}=0 .
\end{aligned}
$$

Therefore, we can calculate the NS properties with the successful RMF parameter set GM1. By resolving equations (7)-(12), we get the total energy density and pressure which can be inputs for solving the Tolman-Oppenheimer-Volkoff (TOV) equation to get the mass-radius relation $[4,5]$.

2.2. NS Cooling Theory with the Protonic ${ }^{1} S_{0}$ Superfluidity. The main contribution to a luminosity of NS core is from the nucleonic direct Urca processes. We calculate NS cooling rate based on the isothermal interior approximation. The heat-balance equation is given by

$$
C_{v} \frac{\mathrm{d} T}{\mathrm{~d} t}=-\left(L_{v}+L_{r}\right)
$$

where $C_{v}$ is the total NS thermal capacity:

$$
C_{v}=c_{e}+c_{\mu}+c_{n}+c_{p} R_{p},
$$

where $R_{p}$ is the heat capacity's inhibiting factor due to the appearance of the protonic ${ }^{1} S_{0}$ superfluids in NS matter [19]:

$$
R_{p}=\frac{3.149 \exp \left(-1.764 T_{\mathrm{cp}} / T\right)}{\left(T / T_{\mathrm{cp}}\right)^{2.5}},
$$

where $T_{\mathrm{cp}}$ is the transition temperature of the protonic ${ }^{1} S_{0}$ superfluids. It is closely related to the protonic pairing gap $\Delta_{p}$ which can be obtained by solving the BCS gap equation at zero temperature approximation $[38,39]$ :

$$
T_{\mathrm{cp}} \doteq 0.66 \times 10^{10} \Delta_{p}
$$

Neutrino and photon luminosities are as follows:

$$
\begin{aligned}
& L_{v}=\int Q e^{2 \Phi} \mathrm{d} V, \\
& L_{r}=4 \pi R^{2} \sigma_{L}(10 T)^{8 / 3} e^{2 \Phi_{s}},
\end{aligned}
$$

where $Q=Q_{0} R_{p 0}$ is the rate of neutrino losses per unit volume for the nucleonic direct Urca processes; $Q_{0}$ is neutrino emissivity without consideration for the protonic ${ }^{1} S_{0}$ superfluidity in NS matter $[40,41] ; R_{p 0}$ is the factor characterizing the suppression of neutrino emissivity due to the protonic ${ }^{1} S_{0}$ superfluidity in NS matter:

$$
R_{p 0}=\frac{0.0163 \exp \left(-1.764 T_{\mathrm{cp}} / T\right)}{\left(T / T_{\mathrm{cp}}\right)^{5.5}} .
$$

In the case without thinking about the protonic ${ }^{1} S_{0}$ superfluids, $R_{p 0}=R_{p}=1$. In equation (17), $e^{\Phi}=\sqrt{1-2 m / r}$ is the gravitational redshift and $R$ is the radius of a NS. In equation (18), $e^{\Phi_{s}}$ expresses the value of $e^{\Phi}$ at the stellar surface (where $r=R$ ). The semiempirical expression $T_{s}=$ $(10 T)^{2 / 3}$ is the relation between the temperature of interior $T$ and temperature of surface $T_{s} . \sigma_{L}$ is the Stefan-Boltzmann constant.

We adopt the Reid soft core (RSC) potential for the pp interaction in the protonic ${ }^{1} S_{0}$ pairings. The detailed description and justification of the method can be seen in references $[42,43]$, and then the theoretical cooling curves with and without $K^{-}$and $\bar{K}^{0}$ condensed phases can be obtained, respectively.

\section{Calculation Results and Discussion}

We start with the simplest components of NSs cores, consisting of $\mathrm{n}, \mathrm{p}$, e, and possibly $\mu$ (recorded as npe $\mu$ matter). For the Bose-Einstein condensed phases, we consider $K^{-}$and $\bar{K}^{0}$ condensations and also discuss a new family of superdense stars with antikaon condensates beyond the NS branch (recorded as npe $\mu K^{-} \bar{K}^{0}$ matter). The strengths of $K^{-}$and $\bar{K}^{0}$ condensations are also sensitively dependent on the optical potential of antikaons $U_{\bar{K}}$ at saturation density of nuclear matter. $U_{\bar{K}}$ is limited to the range of -80 to $-150 \mathrm{MeV}$. The properties of the massive NSs are derived using the successful RMF parameter set GM1 $[33,44]$. The mass range in Figure 1 for the appearance of antikaon condensed phases can help us understand the cooling properties. As shown in Figure 1, the mass ranges are enlarged for antikaon condensations, while the mass is smaller for the deep optical potential of antikaons. $K^{-}$condensed phases appear for the six cases, while $\bar{K}^{0}$ condensed phases occur only in the massive NSs with the optical potential of antikaons of more than $U_{\bar{K}}=-100 \mathrm{MeV}$. The two points from the bottom on the four lines represent the threshold masses of the appearance of $K^{-}$and $\bar{K}^{0}$ condensed phases for the optical potential of antikaons $U_{\bar{K}}=-100$ to $-130 \mathrm{MeV}$, respectively. The mass ranges of $\bar{K}^{0}$ condensed phases are $m=2.201-2.202 M_{\odot}, 2.136-2.141 M_{\odot}, 2.049-2.062 M_{\odot}$, and 1.939-1.960 $M_{\odot}$ for the optical potential of antikaons $U_{\bar{K}}=-100,-110,-120$, and $-130 \mathrm{MeV}$, respectively, which mean that $\bar{K}^{0}$ condensed phases are very difficult to occur in NSs cores compared to $K^{-}$condensed phases.

Figure 2 shows neutrino emissivities of the nucleonic direct Urca processes for the different optical potential depth of antikaons $U_{\bar{K}}$ as a function of the nucleonic density $\rho_{N}$ in 


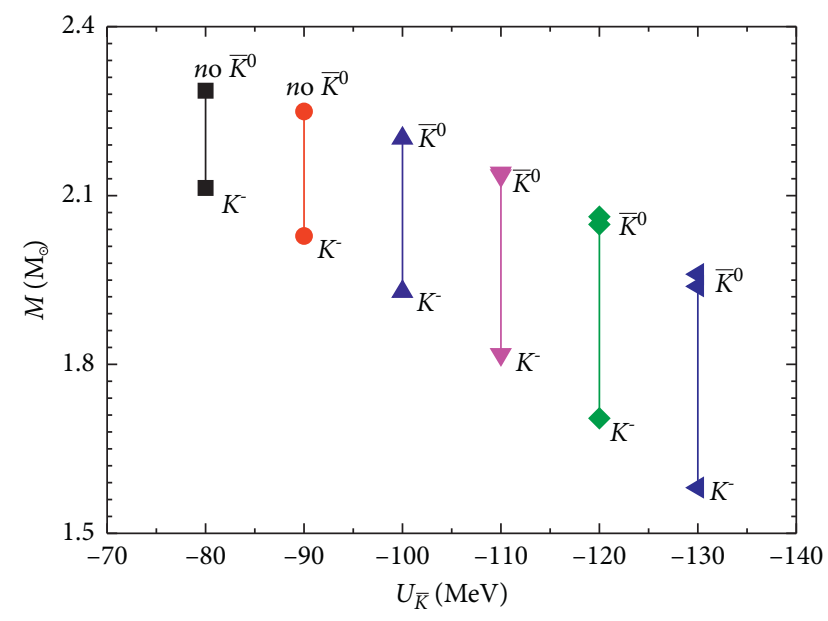

FIGURE 1: Mass ranges of NSs including antikaon condensations as a function of the optical potential of antikaons $U_{\bar{K}}=-80$ to $-130 \mathrm{MeV}$.

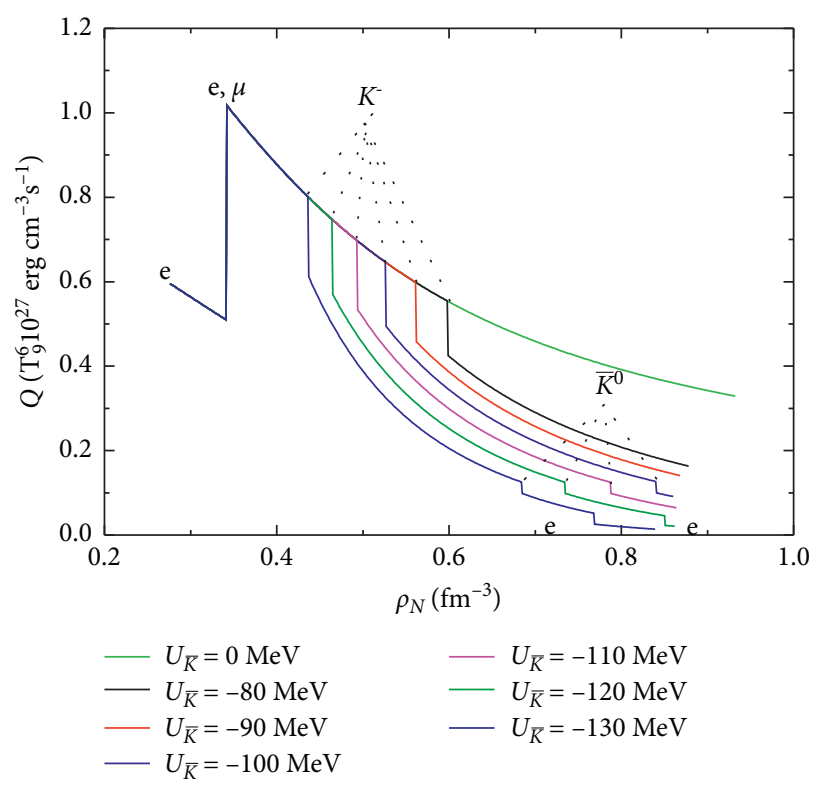

FIGURE 2: Neutrino emissivities of the nucleonic direct Urca processes as a function of the nucleonic density $\rho_{N}$ for the cases of including and not including $K^{-}$and $\bar{K}^{0}$ condensed phases. For comparison, the thick line represents the corresponding values in npe $\mu$ matter.

npe $\mu$ and npe $\mu K^{-} \bar{K}^{0}$ matter. Nucleonic direct Urca processes for $e$ happen at about $\rho_{N}=0.2761 \mathrm{fm}^{-3}$, and then they for $\mu$ occur at about $\rho_{N}=0.3421 \mathrm{fm}^{-3}$ which lead to a steep increase of neutrino energy losses. After that neutrino emissivities of the nucleonic direct Urca processes decline with the growth of the nucleonic densities $\rho_{N}$, and suddenly dip down when $K^{-}$and $\bar{K}^{0}$ condensations appear at a given optical potential of antikaons. This is because the presence of antikaon condensations changes the nucleonic and leptonic fractions. As the optical potential of antikaons gets deeper and deeper, neutrino energy losses of the nucleonic direct Urca processes can occur over a wide interval. Figure 3

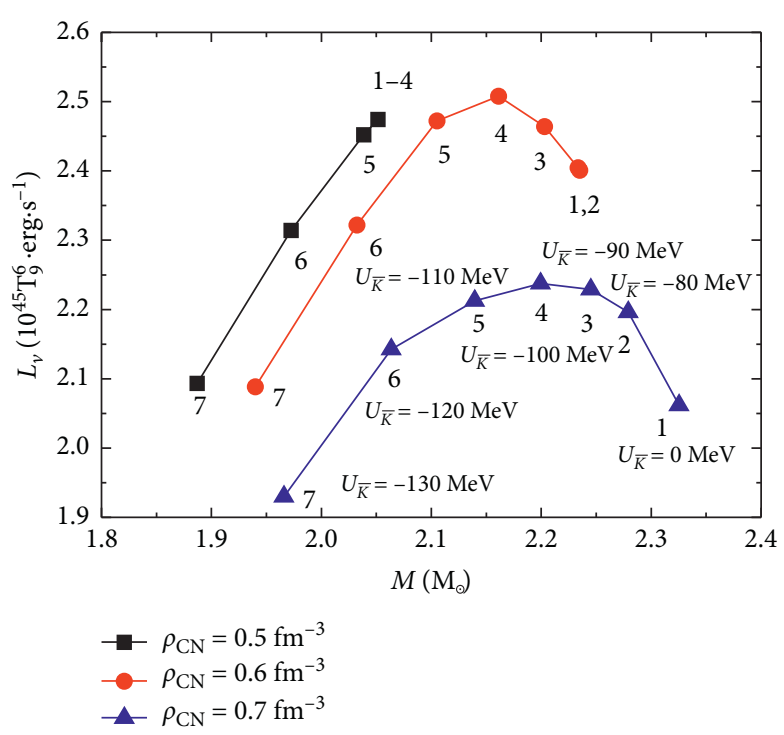

Figure 3: Neutrino luminosities of the nucleonic direct Urca processes as a function of NS mass (M).

presents neutrino luminosities of the nucleonic direct Urca processes with and without $K^{-}$and $\bar{K}^{0}$ condensations as a function of NS mass $M$. Neutrino luminosities from the massive NSs are different for various center densities and NS masses. The values of masses and the corresponding radii are listed in Table 1 for the center densities $\rho_{\mathrm{CN}}=0.5,0.6$, $0.7 \mathrm{fm}^{-3}$ and optical potential of antikaons $U_{\bar{K}}=-80$ to $-130 \mathrm{MeV}$. For the same center densities, the masses are gradually reduced and the corresponding radii grow gradually with the deepening of the optical potential of antikaons, and neutrino luminosities of the nucleonic direct Urca processes decrease for the case of $\rho_{\mathrm{CN}}=0.5 \mathrm{fm}^{-3}$, but first increase and then decrease for the cases of $\rho_{\mathrm{CN}}=0.6$ and $0.7 \mathrm{fm}^{-3}$. In the case of $\rho_{N}=0.5 \mathrm{fm}^{-3}$, no $K^{-}$and $\bar{K}^{0}$ occurred in NSs with the optical potential of antikaons $U_{\bar{K}}=0-100 \mathrm{MeV}$, so that the values of mass, radius, and luminosity are completely same for the potential range. However, for the other two cases, neutrino luminosities increase first because the declines of neutrino emissivities are not enough to make up the growths of NS volumes based on equation (17) and Table 1. The downward tendencies for large potential show that the growths of NS volumes could not compensate the losses for the reduced neutrino emissivities caused by the deep optical potential of antikaons. Furthermore, neutrino luminosities for the same NS mass tend to decrease with the increase of the nucleonic density. For the same increment of the nucleonic densities, the higher the nucleonic densities, the lower the neutrino luminosities.

Neutrino emission is the main cooling mechanism for a NS. Theoretical cooling curves for 12 combinations of the center densities of $\rho_{\mathrm{CN}}=0.5,0.6$, and $0.7 \mathrm{fm}^{-3}$ and optical potential of antikaons of $U_{\bar{K}}=0,-90,-110$, and $-130 \mathrm{MeV}$ for nonsuperfluid NSs are shown in Figure 4, which are almost distinguishable compared with the curve for $1.4 M_{\odot}$ NS without $K^{-}$and $\bar{K}^{0}$ condensed phases. They are obtained through solving the cooling equation (13) on the basis of the 
TABLE 1: Values of NS masses $M$ and the corresponding radii $R$ calculated for the center densities of $\rho_{\mathrm{CN}}=0.5,0.6,0.7 \mathrm{fm}^{-3}$ and optical potential of antikaons $U_{\bar{K}}=0,-80,-90,-100,-110,-120$, and $-130 \mathrm{MeV}$. The critical temperature $T_{\mathrm{cp}}$ of the protonic ${ }^{1} S_{0}$ superfluids is also listed for $U_{\bar{K}}=0$ to $-130 \mathrm{MeV}$ and $\rho_{\mathrm{CN}}=0.5 \mathrm{fm}^{-3}$.

\begin{tabular}{lcccccc}
\hline$U_{\bar{K}}(\mathrm{MeV})$ & 0 & -80 & -90 & -100 & -110 & -120 \\
\hline Case no. & 1 & 2 & 3 & 4 & 5 & -130 \\
\hline$M\left(M_{\odot}, \rho_{\mathrm{CN}}=0.5 \mathrm{fm}^{-3}\right)$ & 2.051 & 2.051 & 2.051 & 2.051 & 2.039 & 1.972 \\
$M\left(M_{\odot}, \rho_{\mathrm{CN}}=0.6 \mathrm{fm}^{-3}\right)$ & 2.235 & 2.233 & 2.203 & 2.161 & 2.105 & 2.032 \\
$M\left(M_{\odot}, \rho_{\mathrm{CN}}=0.7 \mathrm{fm}^{-3}\right)$ & 2.325 & 2.279 & 2.245 & 2.199 & 2.140 & 2.064 \\
$R\left(\mathrm{~km}, \rho_{\mathrm{CN}}=0.5 \mathrm{fm}^{-3}\right)$ & 13.050 & 13.050 & 13.050 & 13.050 & 13.065 & 13.126 \\
$R\left(\mathrm{~km}, \rho_{\mathrm{CN}}=0.6 \mathrm{fm}^{-3}\right)$ & 12.715 & 12.720 & 12.793 & 12.864 & 12.926 & 1.940 \\
$R\left(\mathrm{~km}, \rho_{\mathrm{CN}}=0.7 \mathrm{fm}^{-3}\right)$ & 12.347 & 12.526 & 12.593 & 12.647 & 12.684 & 12.674 \\
$T_{\mathrm{cp}}\left(10^{7} k, \rho_{\mathrm{CN}}=0.5 \mathrm{fm}^{-3}\right)$ & 24.924 & 31.078 & 30.656 & 30.274 & 22.394 & 0.708 \\
\hline
\end{tabular}

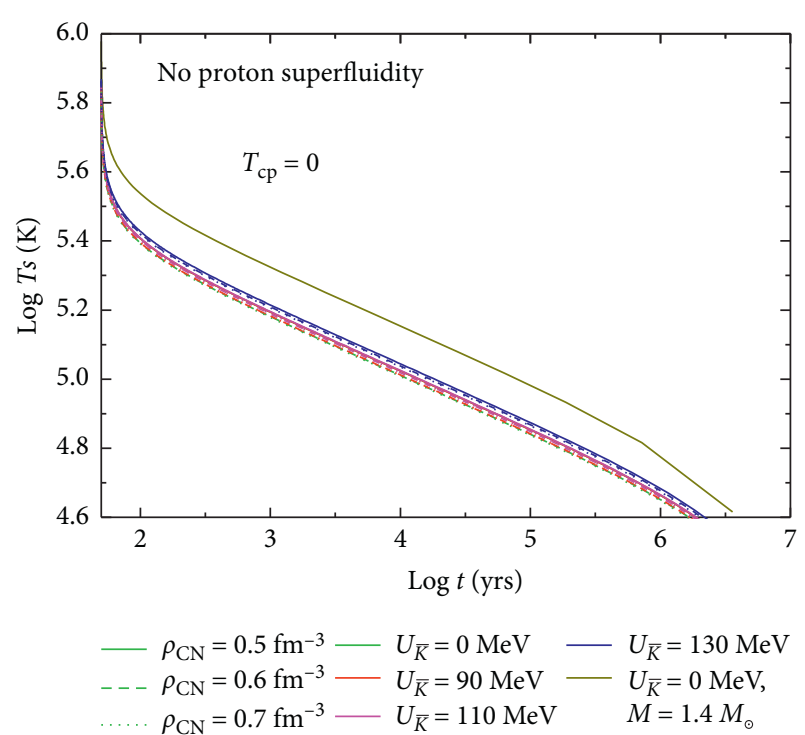

FIgURE 4: Theoretical cooling curves for the nonsuperfluid NS matter compared with the cooling of a nonsuperfluid $1.4 M_{\odot}$ star with $U_{\bar{K}}=0 \mathrm{MeV}$. The initial time $(\mathrm{t})=50$ years.

isothermal interiors approximation of NSs. The minimum mass is $1.887 M_{\odot}$ of 12 combinations with a cooling rate much faster than that of $1.4 M_{\odot}$ NS. Large optical potential of antikaons leads to the mass decrease for a constant center density, but makes NS warmer. Figure 5 shows the theoretical cooling curves of 7 center density $\rho_{\mathrm{CN}}=0.5 \mathrm{fm}^{-3}$ considering and not considering the protonic ${ }^{1} S_{0}$ superfluids, compared with the cooling of a $1.4 M_{\odot}$ star with the critical temperature $T_{\mathrm{cp}}=1.428 \mathrm{~K}$ of the protonic ${ }^{1} S_{0}$ superfluids and without $K^{-}$and $\bar{K}^{0}$ condensed phases. Effective surface emissions from 7 isolated cooling pulsars have been measured and compared with theoretical cooling curves [45]. The values of critical temperature $T_{\mathrm{cp}}$ with the center density $\rho_{\mathrm{CN}}=0.5 \mathrm{fm}^{-3}$ are also listed in Table 1 . As shown in Figure 5, the 7 massive NSs become warmer and slow cooling after the protonic ${ }^{1} S_{0}$ superfluids are considered. Furthermore, the cooling rates of nonsuperfluid NSs slow down for the same center density in Figures 4 and 5, while the cooling rates of NSs with the protonic ${ }^{1} S_{0}$ superfluids speed up for the deep optical potential of antikaons. As a result, the gap between two kinds of theoretical cooling curves with and without the protonic ${ }^{1} S_{0}$ superfluids

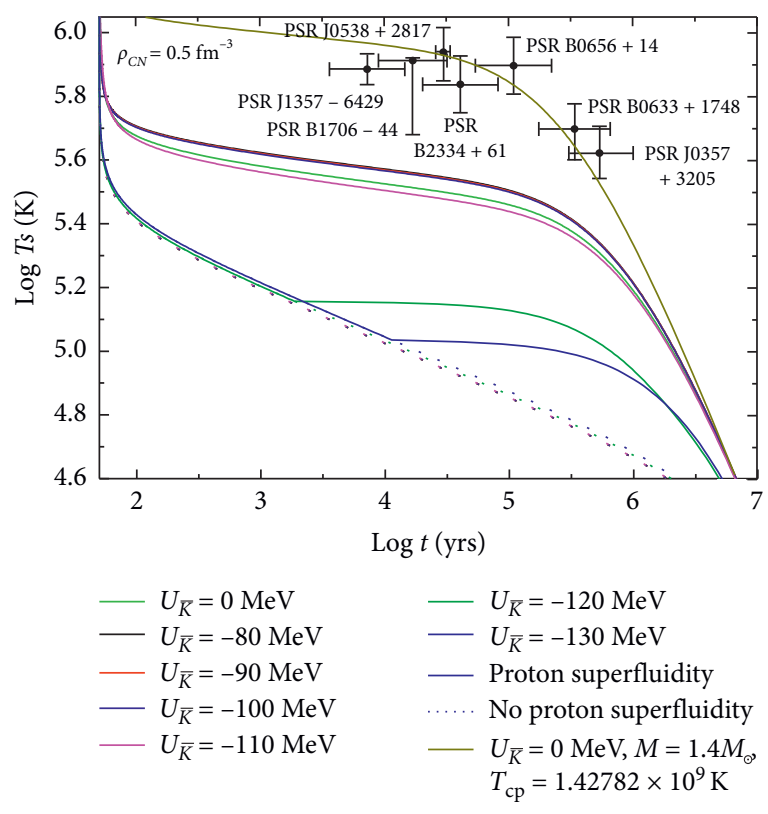

FIgURE 5: Observational data of 7 pulsars J1357-6429, B1706-44, $\mathrm{J} 0538+2817, \quad \mathrm{~B} 2334+61, \quad \mathrm{~B} 0656+14, \quad \mathrm{~B} 0633+1748, \quad$ and J0357 + 3205 [45] compared with the cooling curves including the protonic ${ }^{1} S_{0}$ superfluids models in cores of NSs. Thick line is for the cooling of a superfluid $1.4 \mathrm{M}_{\odot}$ star with $T_{\mathrm{cp}}=1.428 \mathrm{~K}$ and without $K^{-}$and $\bar{K}^{0}$ condensed phases.

is becoming smaller and smaller. However, as shown in Figure 5, the theoretical cooling curves are unable to interpret observational data of 7 NSs whenever the protonic ${ }^{1} S_{0}$ superfluids are considered or not. Particularly, according to Table 1 and Figures 2 and 3, when the center density $\rho_{\mathrm{CN}}=0.5 \mathrm{fm}^{-3}$ and $U_{\bar{K}}=0,-80,-90$, and $-100 \mathrm{MeV}$, the masses, radii, neutrino emissivities, and luminosities are same, as well as no $K^{-}$and $\bar{K}^{0}$ condensations occur for 4 cases. The difference is caused by the different critical temperatures of the protonic ${ }^{1} S_{0}$ superfluids which is also attributed to the differences of the optical potential of antikaons [31]. Similarly, when $U_{\bar{K}}=-110,-120$, and $-130 \mathrm{MeV}, \bar{K}^{0}$ condensed phases still do not appear at the center density $\rho_{\mathrm{CN}}=0.5 \mathrm{fm}^{-3}$. However, $K^{-}$condensations present and cause a series changes of the global properties of NSs. For instance, when the center density $\rho_{\mathrm{CN}}=0.5 \mathrm{fm}^{-3}$, the masses decrease and the corresponding radii increase, critical temperatures of the protonic ${ }^{1} S_{0}$ superfluids, 
neutrino emissivities, and luminosities are gradually reduced for the large optical potential of antikaons (see Figures 2 and 3 and Table 1). These changes result in speedy cooling of the massive NSs due to $\mathrm{K}^{-}$condensations.

\section{Conclusion}

We have studied the cooling properties of the massive NSs by including antikaon condensations in the RMF, BCS, and NS cooling theories. Nucleonic direct Urca processes and the protonic ${ }^{1} S_{0}$ superfluids are considered at neutrino cooling stage of the massive NSs. If the optical potential of antikaons is a constant, neutrino energy losses of the nucleonic direct Urca processes for $e$ and $\mu$ have downward trend as the nucleonic density increases. They decline precipitously when the nucleonic densities equal to the threshold densities of $K^{-}$ and $\bar{K}^{0}$ condensations. When the nucleonic density is a constant, neutrino luminosities of the massive NSs present the trend of decreasing or first increasing and then decreasing when the optical potential of antikaons gets large. If the mass of a NS is a constant, neutrino luminosities also decrease as the nucleonic density is growing. For a constant center density of a nonsuperfluid massive NS, the cooling of the massive NS shows a gradual warming trend with the larger optical potential of antikaons. Yet when the protonic ${ }^{1} S_{0}$ superfluids are considered in a constant center density of a massive NS, they can quicken the NS cooling. In addition, the difference between the cooling rates of them is getting smaller and smaller, following the growth of the optical potential of antikaons. More detailed thermal radiation data about the massive pulsars can help to determine if antikaon condensed phases appear in the cores of the massive pulsars.

\section{Data Availability}

The data are not publicly available due to the restrictions, e.g., their containing information that could compromise the third-party rights and the privacy of research participants.

\section{Conflicts of Interest}

The authors declare that they have no conflicts of interest.

\section{Acknowledgments}

The work was supported by the National Natural Science Foundation of China (no. 11988101, 11805022, and 11803057).

\section{References}

[1] J. Chadwick, "Possible existence of a neutron," Nature, vol. 129 , no. 3252, p. 312, 1932.

[2] W. Baade and F. Zwicky, "Minutes of the stanford meeting," Physical Review, vol. 45, no. 138, p. 1934, 1933.

[3] A. Hewish, S. J. Bell, J. D. H. Pilkington, P. F. Scott, and R. A. Collins, "Observation of A Rapidly pulsating radio source,” Nature, vol. 217, no. 5130, pp. 709-713, 1968.

[4] J. R. Oppenheimer and G. M. Volkoff, "On massive neutron cores,” Physical Review, vol. 55, no. 4, pp. 374-381, 1939.
[5] R. C. Tolman, "Static solutions of einstein's field equations for spheres of fluid," Physical Review, vol. 55, no. 4, pp. 364-373, 1939.

[6] P. B. Demorest, T. Pennucci, S. M. Ransom, M. S. E. Roberts, and J. W. T. Hessels, "A two-solar-mass neutron star measured using Shapiro delay," Nature, vol. 467, no. 7319, pp. 1081-1083, 2010.

[7] J. Antoniadis, P. C. C. Freire, N. Wex et al., "A massive pulsar in a compact relativistic binary," Science, vol. 340 , no. 6131, pp. 448-502, 2013.

[8] H. T. Cromartie, E. Fonseca, S. M. Ransom et al., "Relativistic Shapiro delay measurements of an extremely massive millisecond pulsar," Nature Astronomy, vol. 4, no. 1, pp. 72-76, 2019.

[9] D. D. Ofengeim, "Universal properties of maximum-mass neutron stars: a new tool to explore superdense matter," Physical Review D, vol. 101, p. 103029, 2020.

[10] R. Stabler, "Energy loss mechanisms from very dense stars," Ph. D. thesis, Cornell University, Ithaca, NY, USA, 1960.

[11] H.-Y. Chiu, "Supernovae, neutrinos, and neutron stars," Annals of Physics, vol. 26, no. 3, pp. 364-410, 1964.

[12] H.-Y. Chiu and E. E. Salpeter, "Surface X-ray emission from neutron stars," Physical Review Letters, vol. 12, no. 15, pp. 413-415, 1964.

[13] D. C. Morton, "Neutron stars as X-ray sources," Nature, vol. 201, no. 4926, pp. 1308-1309, 1964.

[14] J. N. Bahcall and R. A. Wolf, "Neutron stars. II. Neutrinocooling and observability," Physical Review B, vol. 140, no. 5, pp. 1452-1466, 1965.

[15] D. Yakovlev, A. D. Kaminker, O. Y. Gnedin et al., "Neutrino emission from neutron stars," Physics Reports, vol. 354, no. 12, pp. 1-155, 2001.

[16] D. G. Yakovlev, O. Y. Gnedin, A. D. Kaminker, K. P. Levenfish, and A. Y. Potekhin, "Neutron star cooling: theoretical aspects and observational constraints," Advances in Space Research, vol. 33, no. 4, pp. 523-530, 2004.

[17] I. Vida, "Short introduction to the physics of neutron stars," EPJ Web of Conferences, vol. 227, p. 01018, 2020.

[18] T. Takatsuka and R. Tamagaki, "Nucleon superfluidity in kaon-condensed neutron stars," Progress of Theoretical Physics, vol. 94, no. 3, pp. 457-461, 1995.

[19] D. G. Yakovlev, K. P. Levenfish, and Y. A. Shibanov, "Cooling of neutron stars and superfluidity in their cores," Physics Uspekhi, vol. 42, no. 8, pp. 737-778, 1999.

[20] D. B. Kaplan and A. E. Nelson, "Strange goings on in dense nucleonic matter," Physics Letters B, vol. 175, no. 1, pp. 57-63, 1986.

[21] N. K. Glendenning and J. Schaffner-Bielich, "First order kaon condensate," Physical Review C, vol. 60, p. 025803, 1999.

[22] S. Pal, D. Bandyopadhyay, and W. Greiner, "Antikaon condensation in neutron stars," Nuclear Physics A, vol. 674, no. 34, pp. 553-577, 2000.

[23] E. E. Kolomeistev and D. N. Voskresensky, "Negative kaons in dense baryonic matter," Physical Review C, vol. 68, no. 1, p. 015803, 2003.

[24] W. B. Ding, G. Z. Liu, M. F. Zhu et al., "K 0 condensation in hyperonic neutron star matter," Chinese Physics Letters, vol. 25, no. 2, pp. 458-461, 2008.

[25] W. B. Ding, G. Z. Liu, M. F. Zhu et al., "The influence of antikaon condensations on neutrino emissivity from neutron stars,", Astronomy \& Astrophysics, vol. 506, pp. 13-16, 2009.

[26] S. Banik, R. Nandi, and D. Bandyopadhyay, "Melting of antikaon condensate in protoneutron stars," Physical Review C, vol. 86, no. 4, Article ID 045803, 2012. 
[27] C. Wu, W.-L. Qian, Y.-G. Ma, and J.-F. Yang, "Neutron stars with kaon condensation in relativistic effective model," International Journal of Modern Physics E, vol. 22, no. 5, p. 1350026, 2013.

[28] N. Gupta and P. Arumugam, "Antikaons in neutron star studied with recent versions of relativistic mean-field models," textitJournal of Physics: Conference Series, vol. 420, Article ID 012154, 2013.

[29] A. Mesquita, M. Razeira, R. Ruffini et al., "An effective field theory for neutron stars with many-body forces, strong $\sum$-repulsion, andK-and \$ $\$$ bar K0 \$ condensation," Astronomische Nachrichten, vol. 336, no. 8-9, pp. 880-884, 2015.

[30] T. Muto, T. Maruyama, T. Tatsumi et al., "Equation of state with kaon condensation and hyperons in dense matter," JPS Conference Proceedings, vol. 20, Article ID 011038, 2018.

[31] Y. Xu, X. L. Huang, X. J. Zhang et al., "The influence of antikaon condensations on nucleon 1S0 superfluidity in neutron star matter," Monthly Notices of the Royal Astronomical Society, vol. 474, no. 3, pp. 3576-3581, 2018.

[32] F. Yang and H. Shen, "Influence of the hadronic equation of state on the hadron-quark phase transition in neutron stars," Physical Review C, vol. 77, no. 2, Article ID 025801, 2008.

[33] T. Miyatsu, M. K. Cheoun, and K. Saito, "Equation of state for neutron stars in SU (3) flavor symmetry," Physical Review C, vol. 88, no. 1, Article ID 015802, 2013.

[34] Y. Xu, Z. Yu, X.-J. Zhang et al., "Direct Urca processes involving proton $1 \mathrm{~S} 0$ superfluidity in neutron star cooling," Communications in Theoretical Physics, vol. 69, no. 4, pp. 425-433, 2018.

[35] Z.-Y. Zhu, E.-P. Zhou, and A. Li, "Neutron star equation of state from the quark level in light of GW170817," The Astrophysical Journal, vol. 862, no. 2, pp. 98-106, 2018.

[36] W. B. Ding, Z. Yu, Y. Xu et al., "Neutrino emission and cooling of dark-matter-admixed neutron stars," Chinese Physics Letters, vol. 36, no. 4, Article ID 049701, 2019.

[37] N. Gupta and P. Arumugam, "Impact of hyperons and antikaons in an extended relativistic mean-field description of neutron stars," Physical Review C, vol. 88, no. 1, Article ID 015803, 2013.

[38] P. Haensel and O. Y. Gnedin, "Direct URCA processes involving hyperons and cooling of neutron stars," Astronomy \& Astrophysics, vol. 290, pp. 458-462, 1994.

[39] D. G. Yakovlev, O. Y. Gnedin, A. D. Kaminker et al., "Theory of cooling neutron stars versus observations," AIP Conference Proceedings, vol. 983, pp. 379-387, 2008.

[40] M. Prakash, M. Prakash, J. M. Lattimer et al., "Rapid cooling of neutron stars by hyperons and delta isobars," Astrophysical Journal Letters, vol. 390, p. 77, 1992.

[41] L. B. Leinson, "Direct Urca processes on nucleons in cooling neutron stars," Nuclear Physics A, vol. 707, no. 3-4, pp. 543-560, 2002.

[42] S. Nishizaki, T. Takatsuka, N. Yahagi, and J. Hiura, "Effective two-nucleon interaction in asymmetric nuclear matter," Progress of Theoretical Physics, vol. 86, no. 4, pp. 853-866, 1991.

[43] Y. Xu, Q. J. Zhi, Y. B. Wang et al., "Nucleonic 1S0 superfluidity induced by a soft pion in neutron star matter with antikaon condensations," Chinese Physics Letters, vol. 36, no. 6, Article ID 061301, 2019.

[44] S. Banik and D. Bandyopadhyay, "Third family of superdense stars in the presence of antikaon condensates," Physical Review $C$, vol. 64, no. 5, Article ID 055805, 2001.
[45] M. V. Beznogov and D. G. Yakovlev, "Statistical theory of thermal evolution of neutron stars," Monthly Notices of the Royal Astronomical Society, vol. 447, pp. 1598-1609, 2005. 